\title{
Association between GDF-15 levels and changes in vascular and physical function in older patients with hypertension
}

\author{
Maryam Barma $^{1,4}$ (D) Faisel Khan ${ }^{1} \cdot$ Rosemary J. G. Price $^{1} \cdot$ Peter T. Donnan $^{2}$ • \\ C. Martina Messow ${ }^{3}$ - Ian Ford ${ }^{3}$ - Alex McConnachie ${ }^{3}$ - Allan D. Struthers ${ }^{1}$. \\ Marion E. T. McMurdo ${ }^{1} \cdot$ Miles D. Witham ${ }^{1}$
}

Received: 1 June 2016/Accepted: 29 September 2016/Published online: 12 October 2016

(c) The Author(s) 2016. This article is published with open access at Springerlink.com

\begin{abstract}
Background Growth differentiation factor-15 (GDF-15) may be a biomarker of disease, protective response and/or prognosis, in older people with hypertension.

Aims To correlate baseline GDF-15 levels with physical and vascular health data in this population.

Methods Baseline blood samples were analysed using a GDF-15 ELISA assay kit. Correlations with baseline and 12-month outcome data, including measures of physical and vascular function, were performed.

Results A total of 147 individuals, mean age $76.8 \pm 4.7$ years, were included. $77(52 \%)$ were male. Baseline $\log _{10}$ GDF-15 showed significant correlations with age $(r=0.37, p<0.001)$, total cholesterol $(r=-0.33$, $p<0.001)$ and 6 -min walking distance $(r=-0.37$, $p<0.001)$. Age remained significantly associated with $\log _{10}$ GDF-15 in multivariable analysis (beta $=-0.29$, $p=0.001$ ). Baseline $\log _{10}$ GDF-15 was significantly associated with decline in 6-min walk distance over 12 months (beta $=-0.27, p=0.01$ ) in multivariable models. No significant correlations were seen with changes in vascular function over 12 months.

Conclusion Baseline GDF-15 predicts declining physical, but not vascular, function in our population.
\end{abstract}

Maryam Barma

m.barma@dundee.ac.uk

1 Division of Cardiovascular and Diabetes Medicine, University of Dundee, Dundee, UK

2 Dundee Epidemiology and Biostatistics Unit, University of Dundee, Dundee, UK

3 Robertson Centre for Biostatistics, University of Glasgow, Glasgow, UK

4 Ageing and Health, Ninewells Hospital, Dundee, UK
Keywords Ageing - Biomarker - Cardiovascular disease · Frailty $\cdot$ Inflammation

\section{Introduction}

The decline in physical function that commonly accompanies ageing is multifactorial in aetiology. Impairments in multiple organ systems, including muscle, nerve, bone, blood vessels and the brain, all contribute to this loss in function. Cardiovascular disease (CVD) in particular is a common cause of hospital admissions amongst older patients and is also a key driver of functional decline. Functional loss, and the accompanying vulnerability to stressors, is captured in the concept of frailty [1]. It is clear that in order to effectively manage frailty, an approach that protects, or bolsters function, across multiple organ systems-i.e. an approach that builds resilience-is necessary [2]. For such an approach to be practical, interventions that target common pathologies affecting multiple organ systems are required; targets such as chronic inflammation, oxidative stress and mitochondrial dysfunction are examples of this. Both CVD and sarcopenia (the age-related decline in muscle function) have been linked to chronic inflammation. Effective interventions may be best deployed early in the process of decline, and to detect those at risk, markers that predict decline before the onset of frailty would be useful.

GDF-15 may represent a potential biomarker of agerelated disease. Discovered in 1997, it is classified structurally as a divergent member of the transforming growth factor $\beta$ (TGF- $\beta$ ) superfamily of cytokines [3]. This family comprises both pro- and anti-inflammatory molecules implicated in diverse inflammatory and fibrotic conditions. Activated macrophages produce GDF-15, and it is believed 
to serve a self-regulatory function by limiting macrophage activation. It is ubiquitously expressed, and levels rise with age, perhaps reflecting its role in regulating cell proliferation, apoptosis and repair, all of which constitute basic cellular stress responses $[4,5]$. It may also affect energy metabolism and storage [6], showing correlations with BMI, inflammatory conditions (e.g. rheumatoid arthritis) and malignancies [7, 8]. A persistent, low-grade pro-inflammatory state is thought to be a key pathophysiological mechanism underlying ageing [9]. Given this, and the potentially protective role that GDF-15 might have in inflammatory regulation (10), it may provide a novel candidate biomarker for predicting functional decline across multiple organ systems.

We therefore tested whether GDF-15 was (a) associated with baseline measures of vascular and physical function and (b) predicted future decline in vascular and physical function, in a cohort of older patients with isolated systolic hypertension.

\section{Methods}

We conducted analyses using samples and data collected prospectively as part of the vitamin $\mathrm{D}$ in isolated systolic hypertension (VitDISH) randomised controlled trial, which tested the effect of vitamin D supplementation on blood pressure in patients aged 70 and over with isolated systolic hypertension [10]. Participants were randomised into VitDISH if they had a baseline systolic BP of $>140 \mathrm{mmHg}$ but $<180 \mathrm{mmHg}$, a baseline diastolic $\mathrm{BP}$ of $<90 \mathrm{mmHg}$ and a baseline 25OHD level of $<75 \mathrm{nmol} / \mathrm{L}$. Participants received 100,000 units of oral vitamin D3 or placebo every 3 months for a year in the trial, which showed no significant effect of vitamin D supplementation on any outcome measured. The trial was approved by the Fife and Forth Valley National Health Service Research Ethics Committee [10].

Outcome measurements from the VitDISH trial available for this analysis were BMI, office systolic and diastolic blood pressure, B-type natriuretic peptide, C-reactive protein, serum-adjusted calcium, glucose, cholesterol, parathyroid hormone and 25OHD levels, submaximal exercise capacity measured using the 6-min walk, hospital anxiety and depression scores (HADS), endothelial function measured using brachial artery flow-mediated dilatation (FMD), and carotid-radial pulse wave velocity using the Sphygmocor system as a measure of arterial stiffness. Baseline demographic data included age, gender and a past medical history of the following conditions: diabetes mellitus, myocardial infarction, angina, peripheral vascular disease and stroke/TIA.
Stored baseline serum samples were used for GDF-15 assays. Samples were allowed to clot at room temperature for $15 \mathrm{~min}$, then spun at $3000 \mathrm{rpm}$ for $10 \mathrm{~min}$ and stored in freezers $\left(-20^{\circ} \mathrm{C}\right)$ for between three and a half to 5 years. GDF-15 was measured in duplicate using a Quantikine ${ }^{\circledR}$ ELISA kit (R\&D Systems, Inc., Minneapolis, MN, USA). The mean intra-assay coefficient of variation $(\mathrm{CV})$ was $4.4 \%$, and the inter-assay CV was $19.8 \%$.

All statistical analyses were performed using IBM SPSS Statistics version 22.0 (IBM, New York, USA). Significance was defined as a two-sided $p<0.05$. Baseline population characteristics are presented as frequencies and proportions, mean values with standard deviations or as medians with inter-quartile ranges (Table 1). As GDF-15, CRP and BNP levels were not normally distributed, results were log-transformed prior to analysis to enable parametric testing. Associations between baseline variables were examined using Pearson's correlation coefficient for continuous variables, and with Student's t-test for dichotomous variable. Multivariable linear regression models were used to determine associations between baseline $\log _{10}$ GDF-15 levels and other baseline variables, and between baseline $\log _{10}$ GDF-15 levels and changes in measures of vascular and physical health between baseline and 12 months. Both unadjusted and adjusted analyses were performed, with adjustment for baseline variables likely to influence either vascular health or physical function. The adjusted models were run both with and without age as a covariate, in case the strong correlation between age and $\log _{10}$ GDF15 masked a potentially important association.

\section{Results}

Sufficient stored baseline samples were available for 147/159 participants, who had been randomised to VitDISH. Analyses were conducted on 146 of thes $\sigma e$, as one sample was excluded due to $>30 \%$ difference between duplicates on GDF-15 testing. Table 1 shows the baseline population characteristics for these individuals, along with baseline associations between each variable and $\log _{10} \mathrm{GDF}$ 15 levels. Baseline $\log _{10}$ GDF-15 levels were significantly associated with increasing age, total cholesterol levels, diastolic BP and HADS depression scores. Independent sample t-tests showed significantly higher baseline $\log _{10-}$ GDF-15 in males, and those with a history of diabetes, myocardial infarction and angina. No significant associations were demonstrated with baseline measures of vascular function. Baseline multivariable linear regression showed GDF-15 levels remained associated only with age (beta $=0.29, p<0.001)$. 
Table 1 Baseline details and univariate associations with GDF-15 levels $(n=146)$ [11]

\begin{tabular}{|c|c|c|c|}
\hline Factor & Baseline level & Correlation versus $\log _{10}$ GDF-15 & $p$ for correlation \\
\hline Mean age (years) (SD) & $76.8(4.7)$ & $0.37 *$ & $<0.001$ \\
\hline Body mass index $\left(\mathrm{Kg} / \mathrm{m}^{2}\right)(\mathrm{SD})$ & $28.2(4.8)$ & 0.03 & 0.69 \\
\hline Median baseline GDF-15 (pg/mL) (IQR) & $400(246)$ & - & - \\
\hline Systolic blood pressure (mmHg) (SD) & $162(11)$ & 0.01 & 0.91 \\
\hline Diastolic blood pressure $(\mathrm{mmHg})(\mathrm{SD})$ & $78(7)$ & $-0.17 *$ & 0.04 \\
\hline Adjusted calcium (mmol/L) (SD) & $2.31(0.07)$ & -0.07 & 0.42 \\
\hline Glucose $(\mathrm{mmol} / \mathrm{L})(\mathrm{SD})$ & $5.3(0.8)$ & 0.10 & 0.26 \\
\hline Total cholesterol (mmol/L) (SD) & $4.9(1.1)$ & $-0.33^{*}$ & $<0.001$ \\
\hline Parathyroid Hormone (pmol/L) (SD) & $5.4(2.5)$ & 0.07 & 0.42 \\
\hline Median C-reactive protein $(\mathrm{mg} / \mathrm{L})(\mathrm{IQR})$ & $1.7(0.8-3.9)$ & 0.04 & 0.63 \\
\hline Median BNP (pg/mL) (IQR) & $31(14-81)$ & 0.11 & 0.18 \\
\hline 25OHD (nmol/L) (SD) & $44(15)$ & -0.11 & 0.17 \\
\hline Pulse wave velocity $(\mathrm{m} / \mathrm{s})(\mathrm{SD})$ & $8.8(1.2)$ & -0.03 & 0.71 \\
\hline FMD of brachial artery (\%) (SD) & $5.2(2.7)$ & 0.06 & 0.51 \\
\hline Mean HADS score (anxiety) (SD) & $4.2(2.7)$ & -0.03 & 0.69 \\
\hline Mean HADS score (depression) (SD) & $3.4(2.4)$ & $0.26^{*}$ & 0.001 \\
\hline \multirow[t]{2}{*}{ Six-minute walking distance (m) (SD) } & $402(99)$ & $-0.37 *$ & $<0.001$ \\
\hline & & $\begin{array}{l}\text { Mean } \log _{10} \mathrm{GDF}-15 \\
\text { Present versus absent }\end{array}$ & $p$ for comparison \\
\hline Male, N. (\%) & $77(52)$ & 2.63 versus $2.56^{*}$ & 0.01 \\
\hline Diabetes mellitus, N. (\%) & $17(12)$ & 2.74 versus $2.58^{*}$ & $<0.001$ \\
\hline Myocardial infarction, N. (\%) & $9(6)$ & 2.74 versus $2.59^{*}$ & 0.02 \\
\hline Angina/PTCA/CABG, N. (\%) & $33(22)$ & 2.66 versus $2.58 *$ & 0.02 \\
\hline Peripheral vascular disease, N. (\%) & $10(7)$ & 2.63 versus 2.60 & 0.66 \\
\hline Stroke/TIA, N. (\%) & $16(11)$ & 2.64 versus 2.59 & 0.33 \\
\hline
\end{tabular}

$B N P$ b-type natriuretic peptide, $C A B G$ coronary artery bypass graft, $F M D$ flow-mediated dilatation $G D F-15$ growth differentiation factor-15, $H A D S$ hospital anxiety and depression score, $H D L$ high-density lipoprotein, $I Q R$ interquartile range, $L D L$ low-density lipoprotein, $P T C A$ percutaneous transluminal coronary angioplasty, $S D$ standard deviation, TIA transient ischaemic attack

$* p<0.05$

Baseline GDF-15 correlated significantly with 12-month changes in LDL cholesterol $(r=0.06, p=0.019)$ and 6-min walk distance $(r=0.23, p=0.009)$, but not with other measures. Multivariable linear regression (Table 2) showed that higher baseline GDF-15 was associated with steeper decline in 6-min walk distance at 12 months after adjustment, but no relationship with changes in blood pressure, nor markers of vascular health, was evident.

\section{Discussion}

Our results suggest that in older patients with hypertension, GDF-15 may be an independent predictor of declining physical function, but does not predict change in vascular function. Whilst our investigation supported previous associations of GDF-15 levels with cardiovascular risk factors and the presence of vascular disease, the responsible mechanisms are not clear, as no correlation was seen between GDF-15 and endothelial function nor arterial stiffness (two key pathophysiological intermediaries for causing vascular disease).

The lack of correlation between GDF-15 and both endothelial function and arterial stiffness may be explained by numerous factors. Firstly, both FMD and PWV measure macrovascular, rather than microvascular, function. The PIVUS study also noted no correlation with brachial artery FMD, but observed significant correlations between GDF15 and endothelium-dependent and endothelium-independent microvascular vasodilatation [11]. Another proposed pathway linking GDF-15 to CVD is the phosphoinositide 3-kinase (PI3k)/protein kinase $\mathrm{C} \zeta(\mathrm{PKC} \zeta)$ /specificity protein 1 (SP1) pathway [12]. This increases ABCA1 gene expression and causing greater macrophage cholesterol 
Table 2 Association of baseline $\log _{10}$ GDF-15 Levels with 12-month changes in vascular and physical health

\begin{tabular}{|c|c|c|c|c|c|c|}
\hline \multirow[t]{2}{*}{ Variable } & \multicolumn{2}{|c|}{ Unadjusted } & \multicolumn{2}{|c|}{ Adjusted model excluding age ${ }^{a}$} & \multicolumn{2}{|c|}{ Adjusted model including age } \\
\hline & Beta & $p$ & Beta & $p$ & Beta & $p$ \\
\hline Systolic blood pressure & 0.02 & 0.87 & -0.11 & 0.30 & -0.11 & 0.28 \\
\hline Diastolic blood pressure & 0.02 & 0.80 & -0.14 & 0.17 & -0.16 & 0.14 \\
\hline Pulse wave velocity & -0.06 & 0.56 & -0.04 & 0.65 & -0.05 & 0.64 \\
\hline FMD of brachial artery & -0.03 & 0.78 & 0.04 & 0.55 & 0.05 & 0.54 \\
\hline B-type natriuretic peptide & 0.10 & 0.43 & 0.06 & 0.75 & 0.05 & 0.78 \\
\hline Six-minute walk distance & -0.15 & 0.11 & $-0.26^{*}$ & 0.02 & $-0.27 *$ & 0.01 \\
\hline
\end{tabular}

$F M D$ flow-mediated dilatation, GDF growth differentiation factor

$* p<0.05$

${ }^{a}$ Adjusted for baseline vascular disease, blood pressure, cholesterol, FMD, PWV, BNP, glucose, calcium, PTH, CRP, 25OHD, 6-min walk and sex

efflux, perhaps limiting foam cell formation to confer atherosclerosis protection. This is substantiated by the correlations between GDF-15 and cholesterol levels evidenced by both our results and those of previous studies $[11,13]$. Finally, the macrovascular disease mechanisms in older people (for example, the relevance of vascular calcification) may not be associated with GDF-15 in the same way as changes seen in previously studied younger cohorts.

GDF-15 is an autocrine inhibitor of inflammation, released in response to pro-inflammatory cytokines such as IL-6 or TNF- $\alpha$ [14]. However, its mechanisms of action remain unclear due to the broad diversity of its functions [14]. Unlike traditional anti-inflammatory pathways, detailed molecular knowledge of GDF-15 signalling remains sparse and deserves further exploration. The previously well-established positive correlation between GDF15 and age was corroborated by our results [13]. Numerous age-related conditions (atherosclerosis, chronic kidney disease, obesity and diabetes, cancer and cognitive impairment) are also associated with elevated GDF-15, making it a clinically relevant biomarker. Gene expression studies on hNAG-18 have shown that GDF-15 may influence age-associated pathology development through mechanisms related to obesity, appetite, insulin sensitivity, energy metabolism and mTOR activity [6]. These complex interactions represent pathways that may be implicated in the development of sarcopenia (reduced muscle strength and functional ability), which can impair physical function in old age. Excessively elevated GDF-15 levels, as seen in chronic inflammatory conditions, cancer and ageing, can cause cachexia. These effects seem to be mediated via neurones (in the hypothalamus and area postrema and nucleus tractus solitarius of the brainstem) that regulate appetite [15], and ERK1 and 2, neuropeptide Y and melanocortin signalling [16], confirming the role of proteinwasting and sarcopenia in contributing to worsening 6-min walking distances.
It is also important to acknowledge the potential effects of pre-existing risk factors and co-morbidities on physical function. Our results showed that those with higher baseline GDF-15 levels were more likely to be male, and have a past medical history of diabetes mellitus, myocardial infarction and/or ischaemic heart disease. Given the large degree of overlap between these demographics and GDF-15 levels, it is currently impossible to extricate the effects of each individual factor. Moreover, the lifestyle factors associated with these conditions (such as lack of exercise, poor diet and smoking), and the restrictions on exercise tolerance and activity levels that accompany conditions such as ischaemic heart disease, are also likely to limit physical function. Despite this assortment of contributory factors, it is possible that GDF-15 may serve as a unifying, objective marker for their cumulative effect on physical function.

\section{Strengths, limitations and future work}

Our analysis is strengthened by the availability of detailed phenotypic data on a range of vascular, physical and psychological measures, and by the availability of 1-year follow-up data on most participants, enabling adjustment for several important confounders.

However, a number of limitations deserve comment. The study population was limited by its size and by all participants being of white ethnicity. We therefore cannot assume that they are representative of the wider elderly population. The age of our cohort and the presence of hypertension inevitably limit the scope for correlation analyses to detect links between measures of vascular function and GDF-15 levels, due to the limited range of these variables. A population-based study would likely increase the range of FMD, PWV and GDF-15 values, enhancing the ability to detect associations. Moreover, a wider panel of dependent variables (including measures of microvascular function and frailty-e.g. weight loss, grip 
strength, sit-stand time and ability to carry out activities of daily living) would be of considerable value in future studies. Studying other clinical outcomes such as time to hospital readmission or mortality data would also be of interest. However, meaningful analysis of such outcomes will require larger study sample sizes and longer follow-up than was available for this analysis.

Knowledge of the biological mechanisms of action of GDF-15 remains incomplete. Future studies should attempt not only to determine whether GDF-15 predicts adverse outcomes in a wider range of older people, but to ascertain whether GDF-15 is part of a causal pathway leading to sarcopenia and dysfunction of other organ systems, whether raised GDF-15 levels actively contribute to protective responses to pathology, or whether raised levels are a bystander marker of other pathological processes (e.g. oxidative stress or other inflammatory signals). Finally, the effect of interventions known to improve physical function (e.g. exercise training) on GDF-15 levels may help answer these questions.

\section{Conclusion}

Baseline GDF-15 may be associated with walk distance and predicts decline in walk distance, but is not associated with vascular function in older patients with hypertension. These findings may enable the development of new ways to detect those at risk of functional decline, as well as suggesting new pathways to target the inflammatory causes and consequences of ageing.

Acknowledgments We thank Mrs Lesley McFarlane and Dr Gwen Kennedy for their assistance and advice with the GDF-15 assay.

Funding The VitDISH trial was funded by Chief Scientist Office, Scottish Government (grant number CZH/4/1100)

\section{Compliance with ethical standards}

Conflict of interest None of the authors report any conflicts of interest.

Statement of human and animal rights The original VitDISH trial was approved by the Fife and Forth Valley National Health Service Research Ethics Committee.

Informed consent Participants gave consent at the time of the trial for blood to be stored for future analysis such as that conducted in this study.

Open Access This article is distributed under the terms of the Creative Commons Attribution 4.0 International License (http://crea tivecommons.org/licenses/by/4.0/), which permits unrestricted use, distribution, and reproduction in any medium, provided you give appropriate credit to the original author(s) and the source, provide a link to the Creative Commons license, and indicate if changes were made.

\section{References}

1. Fried LP, Ferrucci L, Darer J et al (2004) Untangling the concepts of disability, frailty, and comorbidity: implications for improved targeting and care. J Gerontol A Biol Sci Med Sci 59:M255M263

2. Witham M, Sayer A (2015) Biological resilience in older people-a step beyond frailty? Eur Geriatr Med 6:101-102

3. Bootcov MR, Bauskin AR, Valenzuela SM et al (1997) MIC-1, a novel macrophage inhibitory cytokine, is a divergent member of the TGF- $\beta$ superfamily. Proc Natl Acad Sci USA 94:1151411519

4. Wallentin L, Zethelius B, Berglund L, et al (2013) GDF-15 for prognostication of cardiovascular and cancer morbidity and mortality in men. PLoS One 8:e78797. doi: 10.1371/journal.pone. 0078797

5. Schober A, Böttner M, Strelau J et al (2001) Expression of Growth differentiation factor-15/ macrophage inhibitory cytokine-1 (GDF-15/MIC-1) in the perinatal, adult, and injured rat brain. J Comp Neurol 439:32-45

6. Wang X, Chrysovergis K, Kosak J et al (2014) hNAG-1 increases lifespan by regulating energy metabolism and insulin/IGF-1/ mTOR signaling. Aging 6:690-704

7. Wiklund FE, Bennet AM, Magnusson PKE et al (2010) Macrophage inhibitory cytokine-1 (MIC-1/GDF15): a new marker of all-cause mortality. Aging Cell 9:1057-1064

8. Breit SN, Johnen H, Cook AD et al (2011) The TGF-b superfamily cytokine, MIC-1/GDF15: a pleotrophic cytokine with roles in inflammation, cancer and metabolism. Growth Factors 29:187-195

9. Franceschi C, Campisi J (2014) Chronic Inflammation (Inflammaging) and its potential contribution to age-associated diseases. J Gerontol A Biol Sci Med Sci 69(Suppl 1):S4-S9

10. Witham MD, Price RG, Struthers AD et al (2013) Cholecalciferol treatment to reduce blood pressure in older patients with isolated systolic hypertension: the vitdish randomized controlled trial. JAMA Intern Med 173:1672-1679

11. Lind L, Wallentin L, Kempf T et al (2009) Growth-differentiation factor-15 is an independent marker of cardiovascular dysfunction and disease in the elderly: results from the Prospective Investigation of the Vasculature in Uppsala Seniors (PIVUS) Study. Eur Heart J 30:2346-2353

12. Wu J-F, Wang Y, Zhang M et al (2014) Growth differentiation factor-15 induces expression of ATP-binding cassette transporter A1 through $\mathrm{PI} 3-\mathrm{K} / \mathrm{PKC} / \mathrm{SP} 1$ pathway in THP-1 macrophages. Biochem Biophys Res Commun 444:325-331

13. Daniels LB, Clopton P, Laughlin GA et al (2011) Growth-differentiation factor-15 is a robust, independent predictor of 11-year mortality risk in community-dwelling older adults: the Rancho Bernado Study. Circulation 123:2101-2110

14. Corre J, Hébraud B, Bourin P (2013) Concise review: growth differentiation factor 15 in pathology: a clinical role? Stem Cells Transl Med 2:946-952

15. Tsai VW, Husaini Y, Manandhar R et al (2012) Anorex$\mathrm{ia} /$ cachexia of chronic diseases: a role for the TGF- $\beta$ family cytokine MIC-1/GDF15. J Cachexia Sarcopenia Muscle 3:239-243

16. Unsicker K, Spittau B, Krieglstein K (2013) The multiple facets of the TGF- $\beta$ family cytokine growth/differentiation factor15/macrophage inhibitory cytokine-1. Cytokine Growth Factor Rev 24:373-384 